\title{
Calculation of molecular lipophilicity: state of the art and comparison of methods on more than 96000 compounds M Mannhold*1, GI Poda ${ }^{2}$, C Ostermann ${ }^{3}$ and IV Tetko ${ }^{4}$
}

Address: ${ }^{1}$ Düsseldorf, Germany, ${ }^{2}$ Chesterfield, USA, ${ }^{3}$ Konstanz, Germany and ${ }^{4}$ Neuherberg, Germany

* Corresponding author

from 4th German Conference on Chemoinformatics

Goslar, Germany. 9-II November 2008

Published: 5 June 2009

Chemistry Central Journal 2009, 3(Suppl I):O7 doi:I0.II86/I752-I53X-3-SI-O7

This abstract is available from: http://www.journal.chemistrycentral.com/content/3/SI/O7

(C) 2009 Mannhold et al; licensee BioMed Central Ltd.

We first review the state-of-the-art in development of log $P$ prediction approaches falling in two major categories: substructure-based and property-based methods. Then, we compare the predictive power of representative methods for one public $(N=266)$ and two in house datasets from Nycomed $(N=882)$ and Pfizer $(N=95809)$. A total of 30 and 18 methods were tested for public and industrial datasets, respectively. Accuracy of models declined with the number of non-hydrogen atoms. The Arithmetic Average Model (AAM), which predicts the same value (the arithmetic mean) for all compounds, was used as a baseline model for comparison. Methods with Root Mean Squared Error (RMSE) greater than RMSE produced by the AAM were considered as unacceptable. The majority of analyzed methods produced reasonable results for the public dataset but only seven methods were successful on the both in house datasets. We proposed a simple equation based on the number of carbon atoms, $\mathrm{NC}$, and the number of hetero-atoms, NHET: $\log P=1.46( \pm 0.02)+$ $0.11( \pm 0.001) \mathrm{NC}-0.11( \pm 0.001)$ NHET. This equation outperformed a large number of programs benchmarked in this study. Factors influencing the accuracy of log P predictions were elucidated and discussed (the article is in press in J. Pharm. Sci.). 